ज्ञ FRANÇAISE

$\supset \mathrm{DE}$

때 PÉDAGOGIE

\section{Revue française de pédagogie}

Recherches en éducation

174 | janvier-mars 2011

Évaluation, développement professionnel et organisation scolaire

\title{
Conditions d'emploi des auxiliaires de vie scolaire et qualité de l'accompagnement des élèves handicapés
}

Job descriptions of non-medical support workers and quality of service given to disabled students

Condiciones de empleo de los auxiliares de vida escolar y calidad del acompañamiento de los alumnos discapacitados

Einstellungsbedingungen des Hilfspersonals AVS und Qualität der Betreuung der behinderten Schüler

Brigitte Belmont, Éric Plaisance et Aliette Vérillon

\section{OpenEdition \\ Journals}

Édition électronique

URL : http://journals.openedition.org/rfp/2879

DOI : $10.4000 /$ rfp.2879

ISSN : 2105-2913

Éditeur

ENS Éditions

Édition imprimée

Date de publication : 15 mars 2011

Pagination : 91-106

ISBN : 978-2-7342-1203-4

ISSN : 0556-7807

\section{Référence électronique}

Brigitte Belmont, Éric Plaisance et Aliette Vérillon, « Conditions d'emploi des auxiliaires de vie scolaire et qualité de l'accompagnement des élèves handicapés ", Revue française de pédagogie [En ligne], 174 | janvier-mars 2011, mis en ligne le 15 mars 2015, consulté le 19 avril 2019. URL : http:// journals.openedition.org/rfp/2879; DOI : 10.4000/rfp.2879 


\title{
Varia
}

\section{Conditions d'emploi des auxiliaires de vie scolaire et qualité de l'accompagnement des élèves handicapés}

\author{
Brigitte Belmont, Éric Plaisance et Aliette Vérillon
}

Les auxiliaires de vie scolaire sont chargés de l'accompagnement d'élèves handicapés en milieu scolaire ordinaire. Leur mission s'inscrit ainsi dans les processus de division du travail éducatif au sein de l'école et leur nombre en augmentation constante atteste de la reconnaissance par les pouvoirs publics du bien-fondé de cette fonction. Cependant le statut attribué à ces emplois (précarité, faible rémunération, formation courte) conduit à s'interroger sur la possibilité d'assurer, dans ces conditions, des services de qualité et soulève la question de la professionnalisation de ces personnels. La recherche présentée dans cet article a pour objet d'étudier les relations, perçues par les auxiliaires de vie scolaire eux-mêmes, entre leurs conditions d'emploi et l'exercice concret de leur fonction dans les établissements où ils sont nommés. Des entretiens individuels ont été réalisés auprès de 34 auxiliaires de vie scolaire en poste dans le département de Seine-Saint-Denis, en région parisienne. L'enquête permet de restituer leur expérience propre, leurs représentations des rapports entre leur emploi et les activités qu'ils mènent, entre leur statut et leurs engagements sur le terrain. L'analyse montre que le statut qui leur est réservé ne leur semble pas être à la hauteur de la fonction attendue, qui implique un fort engagement personnel et sollicite des compétences diverses. La formation reçue est aussi très critiquée au regard de ce qu'ils en attendent. Sont également présentés les apports et les difficultés de collaboration avec les partenaires, notamment les enseignants et les professionnels spécialisés.

Descripteurs (TESE) : éducation des jeunes à besoins éducatifs particuliers, handicap, enseignant spécialiste, approche inclusive, relations interpersonnelles, coopération.

\section{INTRODUCTION}

La scolarisation des élèves en situation de handicap à l'école ordinaire est une orientation majeure de la loi de 2005 sur les personnes handicapées ${ }^{1}$, bien que le projet personnalisé de scolarisation de certains de ces élèves puisse permettre des modalités de formation extérieures au milieu ordinaire. De fait, la demande, en particulier celle émanant des parents, de scolarisation et de formation la plus proche possible des lieux ordinaires est un phénomène qui s'est développé fortement au cours des années quatre-vingt-dix, puis des années deux mille, au nom de la revendication des droits pour tous. Parallèlement, des moyens d'aide ont 
été réclamés pour permettre aux établissements et à leurs personnels d'assumer cette nouvelle responsabilité d'accueil. La création des auxiliaires de vie scolaire (AVS par la suite) a été une des réponses, en termes de ressources humaines, apportées à cette demande. Leur mission est essentiellement d'assurer un accompagnement de ces élèves dans les actes de la vie quotidienne et de faciliter leur autonomie dans les situations d'apprentissage? ${ }^{2}$.

Le nombre de ces emplois créés, en augmentation constante $^{3}$, atteste de la reconnaissance par les pouvoirs publics du bien-fondé de cette fonction qui s'appuie sur un certain consensus sociopolitique, au moins sur le plan du principe de l'accompagnement scolaire. Ainsi la Commission nationale consultative des droits de l'Homme (2008, p. 7) énonce : « Les AVS sont une condition nécessaire pour atteindre les objectifs de la loi du 11 février 2005 ». En réalité, ces emplois ont été créés dès la fin des années quatrevingt à l'initiative de parents et de militants associatifs, mais ils se sont inscrits très tôt dans le cadre des politiques publiques en faveur de l'emploi des jeunes : en 1997, c'est la possibilité de recrutement par le moyen des « emplois jeunes »; puis en 2003, la prise en charge complète (financement, recrutement, formation) par l'Éducation nationale de ceux qui sont alors appelés " assistants d'éducation » et dont une partie d'entre eux a eu la tâche d'accompagner des élèves en situation de handicap ; enfin, depuis octobre 2009, le recours partiel à des associations ou même à des services à domicile pour assumer la responsabilité de certains AVS qui arrivent en fin de contrat, ceci afin de permettre la continuité de l'accompagnement scolaire des enfants handicapés. Certaines associations y voient le signe d'un nouveau désengagement de l'État, malgré les intentions affichées d'assurer la nécessaire pérennité des accompagnements scolaires des enfants concernés. De tels va-et-vient sont le signe des ambiguïtés portant sur le statut, la formation et la rémunération des AVS. Ce sont des jeunes recrutés sur des emplois précaires (3 ans renouvelables, d'année en année, jusqu'à 6 ans), faiblement rétribués et dont la formation obligatoire se limite à 60 heures, dites "d'adaptation à l'emploi ", au cours de la première année. Ce type de précarité a même été renforcé depuis 2005 par le recrutement, dans des conditions encore plus difficiles, des emplois vie scolaire (EVS) relevant d'autres types de contrats (contrats d'avenir ou contrats d'accompagnement dans l'emploi) et destinés à des personnes de niveau CAP ou BEP des filières sanitaires et sociales. On distingue aussi officiellement les AVS selon qu'ils exercent leurs fonctions soit par un suivi individualisé des élèves en situation de handicap dans des classes ordinaires (ce sont les AVSi, mais c'est aussi le cas des EVS), soit par une aide au fonctionnement de dispositifs collectifs, dirigés par des enseignants spécialisés et accueillant des élèves en situation de handicap (ce sont les AVSco). De tels dispositifs collectifs sont actuellement les classes pour l'inclusion scolaire (CLIS) dans l'enseignement du premier degré et les unités localisées pour l'inclusion scolaire (ULIS) dans l'enseignement du second degré. En réalité, au moment de l'enquête, les premiers dispositifs s'appelaient classes d'intégration scolaire (CLIS) et les seconds unités pédagogiques d'intégration (UPI).

Pour contribuer à éclairer le débat social sur les AVS (leur création, leur développement, les enjeux actuels d'un éventuel métier), nous nous sommes intéressés au point de vue des personnes les plus directement concernées par cette question, c'est-àdire les AVS eux-mêmes. Cette approche conduit en premier lieu à s'interroger sur le cadre dans lequel cette fonction a été envisagée par l'institution scolaire et sur les conditions de travail qui ont été proposées pour l'accomplir.

\section{LA FONCTION D'ACCOMPAGNEMENT DES AVS ET LA DIVISION DU TRAVAIL ÉDUCATIF}

La problématique de la division du travail éducatif est à la croisée de la sociologie de l'éducation et de la sociologie du travail. Elle est particulièrement justifiée dès lors que l'on observe les transformations des activités pédagogiques dans le cadre de l'école primaire depuis le milieu des années soixante, dans la mesure où la diversification des tâches et la multiplication des personnels spécialisés se sont développées au nom de l'attention spécifique à accorder aux élèves présentant diverses difficultés. En effet, alors que la longue tradition de l'école primaire était la présence d'un seul maître généraliste face à sa classe, la nécessité d'une approche spécifique des élèves perçus comme différents est apparue. Comme le formule Philippe Perrenoud (1993, p. 37) qui traite de cette situation à Genève, "la division du travail se présente alors comme une réponse possible à la complexité ». Dans le cas français, il est vrai que des classes spécialisées ont été officiellement créées dès la loi de 1909 sur les écoles autonomes et les classes de perfectionnement, pour des enfants considérés comme "arriérés ». Mais ces classes ne se sont 
développées qu'à partir de la fin des années cinquante et surtout dans les années soixante, en n'entamant pas fondamentalement l'hégémonie du modèle du maître généraliste. Les classes de perfectionnement formaient un monde à part (Hugon, 1984). II en va tout autrement dès lors que se multiplient dans les années soixante les formations d'instituteurs spécialisées, qui s'appuient sur des catégorisations nouvelles d'enfants dits " inadaptés » et sur la perspective d'actions différenciées en liaison avec les classes ordinaires, et parfois même au sein de ces classes. L'extension de la spécialisation pour des maîtres issus du corps des instituteurs (rééducateurs et psychologues) a permis des modes d'exercice professionnel qui, non seulement se sont séparés de l'exercice direct de la classe, mais se sont aussi calqués sur le modèle de métiers extra-scolaires, essentiellement pratiqués jusqu'alors en milieu médical ou paramédical. Aujourd'hui, de fortes suppressions de postes de ces personnels, par exemple ceux des réseaux d'aide spécialisés aux élèves en difficulté (RASED), ont été officiellement effectuées, mais elles semblent l'avoir été beaucoup plus pour des raisons d'économies budgétaires que pour renoncer radicalement à la diversification des tâches au sein des écoles, qui peut prendre d'autres formes.

La division du travail éducatif au sein des établissements scolaires primaires reste bien d'actualité lorsqu'il est question de développer l'accueil des élèves handicapés en milieu ordinaire, selon les orientations de la loi de $2005^{4}$. La fonction d'auxiliaire de vie scolaire, qui ne se confond nullement avec celle d'un enseignant spécialisé, y apparaît suscitée par les besoins d'accompagnement de certains enfants reconnus handicapés et dont ne pourrait se charger un enseignant seul, tout au moins dans un certain nombre de cas. Ces cas font d'ailleurs l'objet d'évaluations des besoins d'accompagnement. Cette fonction apparaît relativement complexe et subtile, et plusieurs auteurs l'ont déjà analysée. Puig (2005) distingue " une dimension affective de réassurance ", " une dimension sociale de médiation " et « une dimension technique d'assistance ". Pour LaurentCognet (2005), les AVS peuvent aussi avoir à gérer des problèmes déontologiques, par exemple lorsqu'ils participent à des réunions où sont abordées des informations relevant du secret professionnel. C'est bien la notion d'“ accompagnement » qui est au cœur de la définition de la fonction. Une notion qui est loin d'être propre au travail d'AVS puisqu'elle concerne aussi de larges secteurs d'intervention dans le domaine médico-social, tout en restant généralement floue dans ses contours d'application et dans ses définitions (Stiker, Puig \& Huet, 2009). On constate toutefois, malgré ces incertitudes notionnelles et même malgré les connotations parfois religieuses (comme «partager le pain »), que l'accompagnement prend place dans les orientations actuelles qui veulent favoriser le " projet de vie » des personnes handicapées et leur autonomie dans des milieux le plus possible ouverts et non ségrégatifs ${ }^{5}$. De manière générale, l'engouement pour la notion d'accompagnement témoigne de la tendance à la personnalisation des aides (en lieu et place des dispositifs exclusivement collectifs), voire d'une "individualisation du social » (Mazereau, 2005).

Dans le contexte scolaire, l'accompagnement prend une coloration spécifique, du fait que le professeur reste le seul responsable de la classe, voire le seul «technicien de la pédagogie » (Philip, 2009). De fait, c'est bien une fonction nouvelle qui apparaît attribuée aux AVS (non issus du corps enseignant), renforçant le phénomène de division du travail éducatif mais nécessitant aussi des cohésions d'actions entre les différents intervenants. C'est ainsi que les analyses d'Ebersold (2003) soulèvent la question de la qualité du travail des auxiliaires en fonction de la cohérence des relations qui s'instaurent entre les acteurs d'un projet visant à répondre aux besoins d'un enfant. Le travail d'accompagnement accompli par les AVS nécessiterait alors une " logique d'action commune » (Ebersold, 2003, p. 19).

Se trouvent donc posés conjointement les questions relatives à la place spécifique des AVS dans le cadre scolaire et à leurs relations avec les enseignants, et celles des rapports entre la qualité de leur emploi et la qualité des services qu'ils rendent. Ces dernières questions ne sont pas non plus des questions spécifiques aux AVS (et aux EVS), car elles rejoignent étroitement les interrogations soulevées actuellement à propos de l'ensemble des personnels dédiés aux "services à la personne ", plus précisément à l'égard des personnes en situation de handicap ou des personnes âgées. De nombreux auteurs soulignent la grande contradiction entre, d'une part, la demande de prestations authentiquement professionnelles, attentives aux besoins des personnes et, d'autre part, la médiocrité du statut accordé aux " aidants " : ce sont des salariés « dans une situation de grande fragilité économique et sociale " (Devetter, Jany-Catrice \& Ribault, 2009, p. 5). En d'autres termes, la fonction est unanimement reconnue comme indispensable mais les conditions de sa réalisation par des personnels précaires restent en deçà des exigences d'une professionnalisation ${ }^{6}$. C'est pré- 
cisément cette notion de «professionnalisation » qui est au cœur des débats et des revendications sur le statut des AVS. De manière générale, plusieurs dimensions de la notion sont repérables, le plus souvent en liaison étroite les unes avec les autres, et contribuant ainsi à des usages sociaux entremêlés, voire souvent équivoques (Bourdoncle, 2000). Mais dans le cas présent, la professionnalisation concerne prioritairement l'activité exercée : il s'agit de passer d'une activité mal reconnue, mal rémunérée, accomplie dans un temps limité, au statut incertain, à une activité qui serait socialement organisée et reconnue dans un espace social de référence, qui reposerait sur " un système de règles octroyant un statut à ceux qui exercent l'activité et indiquant des conditions d'accès, notamment en termes de formation " (Cadet, 2008 , p. 340). À propos des «emplois jeunes ", Raymond Bourdoncle (2000) s'était déjà interrogé sur les éventuels débuts de professionnalisation de leurs activités mais il soulignait les difficultés à les pérenniser sous forme de métier stable, du fait d'une activité trop morcelée et peu visible. À propos des assistantes maternelles ${ }^{7}$, François Aballea (2005) a aussi montré un processus « inachevé » de professionnalisation, ou " faiblement entamé ", et ceci malgré différents textes législatifs et réglementaires : l'assistance maternelle resterait encore de l'ordre d'" une opportunité que l'on saisit à un moment de sa biographie " (Aballea, 2005, p. 65). Nous faisons l'hypothèse que les AVS sont eux-mêmes dans cette situation de professionnalisation inachevée.

Compte tenu de l'ensemble de ces analyses, la recherche présentée ici a pour objet d'étudier les relations entre les conditions d'emploi des AVS, caractérisées par un statut de précarité, et l'exercice concret de leurs fonctions dans les écoles où ils sont nommés. Pour ce faire, nous n'avons pas opté pour des analyses de pratiques observées, comme celles qui s'attachent à caractériser différents modes de fonctionnement entre enseignant et AVS (voir par exemple Toullec-Théry \& Nédélec-Trohel, 2008). En faisant appel à leur expérience propre, nous avons plutôt cherché à cerner la façon dont ces nouveaux acteurs conçoivent leur mission dans le système éducatif et les conditions de travail qui leur apparaissent nécessaires pour remplir cette mission. Ce sont ainsi leurs représentations que nous restituons ici : comment se situent-ils par rapport à leur activité, comment la définissent-ils ? Quels sont les rapports entre leur fonction et les tâches qu'ils assurent, entre leur statut et leurs engagements sur le terrain, entre leurs aspirations et leurs relations quotidiennement vécues? Leurs attentes se traduisent-elles en termes de professionnalisation et quelle interprétation en donnent-ils alors?

\section{MÉTHODOLOGIE D'ENQUÊTE}

Une recherche préalable a été menée par questionnaire auprès des AVS et des EVS en fonction en 2006 dans le département de Seine-Saint-Denis. II s'agissait alors d'établir un état des lieux de leurs conditions de travail (Belmont, Plaisance \& Vérillon, 2009). Les principaux résultats de cette enquête, établis à partir des 151 questionnaires reçus, ont fait apparaître que :

- les AVS recrutés, dans leur grande majorité (85\%), projettent de s'engager professionnellement dans le domaine de l'éducation ou du social, ce qui laisse penser que le choix de cet emploi répond, le plus souvent, à une motivation en lien avec la nature du travail proposé ;

- ils reçoivent assez massivement (pour $70 \%$ d'entre eux) une formation d'« adaptation à l'emploi », mais rarement plus d'une semaine ;

- tous les AVS n'ont pas les mêmes possibilités de profiter d'échanges professionnels pour développer leurs compétences. Les AVSi qui ont pour mission d'assurer un suivi individualisé des élèves en situation de handicap), de même que les EVS, suivent en général plusieurs enfants dans plusieurs établissements, et ont moins que les AVSco (qui apportent leur aide dans le cadre de dispositifs collectifs) des contacts avec d'autres partenaires que les enseignants d'accueil, tels que d'autres enseignants, les directeurs et les professionnels spécialisés.

C'est la deuxième étape de la recherche qui fait l'objet de cet article. Elle a été réalisée à partir d'entretiens individuels, semi-directifs centrés, menés auprès de 34 AVS, parmi ceux qui avaient déjà été sollicités pour répondre au questionnaire. Les personnes interrogées ont été sélectionnées en collaboration avec l'équipe de l'inspection académique responsable de la gestion de ces personnels, de façon à constituer un échantillon comportant les différents cas de figure, selon le type de personnels (AVSi, AVS ${ }^{8}{ }^{8}$ ), le type de difficultés des élèves (troubles psychologiques ou cognitifs, déficience motrice, auditive ou visuelle) ou le niveau de scolarité (école maternelle, école élémentaire, collège, lycée). Le guide d'entretien visait à recueillir leur point de vue sur la place qu'ils attribuent à cet emploi dans leur 
parcours professionnel (motivations, perspectives, appréciation portée sur cette expérience) ; sur les modalités de leur travail d'accompagnement auprès des enfants, leurs relations avec les différents partenaires, les difficultés rencontrées ; sur les compétences professionnelles qu'ils jugent nécessaires pour remplir leur fonction, les moyens possibles pour les acquérir, notamment la formation reçue et les besoins ressentis. Afin de dépasser les considérations générales ou le discours convenu, nous avons veillé à les inciter à appuyer leurs propos sur des faits précis, à se référer à des situations concrètes, à expliciter leurs modes d'intervention à partir d'exemples d'enfants suivis. Les entretiens se sont déroulés pour la plupart dans l'établissement scolaire, selon la préférence des personnes interrogées (nous leur avons laissé le choix du lieu de rencontre), dans une salle réservée à cet effet. Cette situation a été I'occasion de prélever des éléments d'information nous permettant de situer les propos recueillis dans leur propre contexte de travail, tel que nous avons pu l'appréhender lors de notre venue. La transcription intégrale des entretiens a ensuite fait l'objet d'une analyse thématique.

\section{ÊTRE AVS, UN FORT INVESTISSEMENT MALGRÉ UN STATUT CONTESTÉ}

Pour les personnes interrogées, le choix d'être AVS apparaît motivé par un projet professionnel qui, comme cela se dessinait dans l'enquête par questionnaire, est envisagé le plus souvent dans le champ de l'éducation. Certains ont un projet bien défini. L'emploi d'AVS est considéré comme un moyen de se préparer au métier visé (notamment enseignant ou éducateur spécialisé). Pour d'autres, cette voie est envisagée comme une réorientation par rapport à des études ou des situations professionnelles qui ne les satisfont pas. Être AVS constitue une première expérience dans le domaine de l'éducation. Comme nous en faisions l'hypothèse, la place que tient cet emploi dans leurs perspectives professionnelles s'avère effectivement susciter un fort investissement. Les entretiens rendent largement compte de ce que cette expérience leur apporte, notamment l'occasion d'une pratique avec les enfants, une découverte des enfants handicapés, de leurs difficultés et capacités de progrès, des connaissances sur le fonctionnement scolaire. Elle les a en général confortés dans leurs choix : « Ça me donne encore plus envie d'être enseignante »; " Je me retrouve dans ce que je voulais faire à la base. » Elle a conduit certains d'entre eux à préciser leur projet (par exemple, enseigner en maternelle) ou leur a ouvert de nouvelles perspectives : "Par rapport au handicap, je me suis découvert une vocation. »

Leur investissement par rapport à cet emploi tient non seulement à la préoccupation de mener à terme un projet professionnel, mais également à la nature du travail lui-même, qui correspond à leurs centres d'intérêt. Leur engagement est associé à l'évocation d'un vécu de satisfaction et de gratification personnelle : " Je trouve ça très bien [...] on travaille avec les élèves, avec le professeur spécialisé, avec les autres professeurs du collège "; "Les progrès qu'il a fait, on se rend compte que c'est grâce à nous aussi. " Leur investissement est de plus sous-tendu par une position éthique par rapport aux enfants handicapés, défendant l'importance de leur apporter une éducation et l'intérêt de leur scolarisation en milieu ordinaire. L'intérêt qu'ils trouvent dans ce travail est particulièrement manifeste chez ceux qui sont AVS depuis deux ou trois ans et ceux qui sont prêts à poursuivre l'année suivante. Certains d'entre eux souhaiteraient même demeurer AVS, si ces emplois étaient pérennisés et devenaient un vrai métier.

La question du statut qui leur est attribué fait partie des thèmes abordés spontanément par les AVS interrogés. Les points de vue exprimés sont toujours critiques. La non-reconnaissance de l'emploi d'AVS comme un métier spécifique est vécue comme une dévalorisation, tant de la fonction que des personnes qui l'assurent : «On fait un métier, mais ce n'est pas un métier, on nous dit: "C'est un service". [...] je ne suis reconnue que par l'institutrice spécialisée, mais pour les autres je ne fais rien. "Au regard de leur expérience, le fait de s'occuper, dans le cadre de l'école, d'élèves handicapés ne peut être assimilé à un simple service que quiconque pourrait effectuer: "Ce n'est pas n'importe quel poste, auxiliaire de vie scolaire. [...] c'est quelque chose qui demande un fort investissement et professionnel et personnel. C'est pour ça que j'ai du mal à parler d'autre chose que de métier ou de profession. " Le caractère temporaire de l'emploi ne leur semble pas favoriser l'investissement qui est nécessaire pour être à la hauteur des attentes. Ils ont l'impression d'être utilisés puis renvoyés, sans considération pour le travail effectué et sans prise en compte des compétences qu'ils ont pu acquérir progressivement : "Quand on sait que c'est provisoire, il y a une espèce d' "à quoi bon". À quoi bon faire tout ça ? De toute façon, on n'est pas reconnu et à la fin du contrat, on nous dira au 
revoir, et puis c'est tout. Et on en reprendra d'autres et on recommencera. »

D'autres problèmes sont encore signalés. Concernant les AVSi, qui suivent généralement plusieurs enfants, l'échelonnement des procédures de décisions relatives aux besoins d'accompagnement des élèves entraîne des modifications d'emploi du temps. Ils peuvent ainsi être conduits de façon impromptue à changer d'école en cours d'année, ce qui les place dans des situations inconfortables, en particulier pour développer des collaborations avec des enseignants : "Les premiers contacts ont été très durs, parce que... Je suis arrivée, il y avait déjà quelqu'un qui s'occupait de la petite. [...] C'est sûr que l'enseignante n'était pas contente, parce qu'on commence un travail avec une personne et puis on change. " Plusieurs AVS évoquent les témoignages de collègues qui se sont trouvés en difficulté et qui parfois même ont abandonné. L'enquête ayant eu lieu au dernier trimestre, les AVS interrogés font partie de ceux qui ont persévéré au moins une année scolaire. $\mathrm{Si}$, en dépit des différents problèmes qu'ils dénoncent, ils se sont impliqués dans ce travail, c'est en raison de leur motivation pour une fonction qu'ils estiment importante pour les enfants : "Malgré le caractère provisoire, malgré la paye qui n'est pas terrible, [si] on s'investit à fond, c'est pour les gamins. " C'est également qu'ils se sont trouvés, contrairement à d'autres, dans des conditions de travail qu'ils jugent plutôt favorables, bénéficiant notamment sur le terrain de la reconnaissance nécessaire qui leur fait défaut sur le plan institutionnel : «On est accepté ici, on est reconnu »; "Je suis bien intégré dans l'équipe pédagogique ". Si l'investissement dans le travail peut être considéré comme propice à l'acquisition des aptitudes requises, les AVS estiment que leurs conditions statutaires actuelles ne sont pas en mesure de soutenir cet investissement, qui repose plutôt sur leurs motivations personnelles.

\section{UN EMPLOI QUI SOLLICITE DES COMPÉTENCES MULTIPLES}

\section{Des dispositions personnelles}

Lorsque les AVS sont interrogés sur les compétences qui leur paraissent nécessaires pour mener à bien leur travail, au-delà de la motivation, ils évoquent en premier lieu ce qui serait " plutôt des qualités humaines " qui les prédisposeraient à assumer ces fonctions. Le plus souvent, ils mentionnent :
- la tolérance, la bienveillance à développer à l'égard d'enfants dont la progression est entravée par des difficultés diverses, la patience indispensable pour ne pas se décourager devant des progrès lents ou incertains ;

- l'écoute, l'attention, la disponibilité nécessaires pour essayer de comprendre ces difficultés ;

- l'adaptabilité dont ils doivent faire preuve pour s'ajuster à chaque enfant, ainsi qu'aux différents enseignants et aux différents établissements dans lesquels ils interviennent ;

- le dynamisme, l'inventivité qu'ils ont à mobiliser pour chercher des moyens d'action efficaces.

\section{Des savoirs et des savoir-faire}

Ils évoquent cependant des savoir-faire professionnels et des connaissances qu'il leur apparaît nécessaire d'acquérir pour effectuer le travail demandé :

- des compétences relationnelles, comme savoir « dialoguer » avec les enfants, savoir « composer avec les différentes personnalités des élèves et des professeurs, et du personnel qui est aux alentours ", ainsi qu'avec les parents ;

- des compétences en lien avec la fonction d'accompagnement, comme savoir trouver la bonne distance entre relations affectives et relations de travail, l'équilibre entre aider les enfants et les laisser développer leur autonomie, un juste niveau d'exigence qui tienne compte de leurs difficultés, "les pousser le plus possible sans aller au-delà de leurs possibilités ${ }^{9} »$;

- un minimum de savoirs scolaires dans les disciplines enseignées, mais aussi des compétences qui relèvent d'une approche pédagogique, comme adopter une attitude éducative aidante (« encourager », « stimuler », " rassurer "), savoir " observer » les enfants, " comprendre leurs difficultés » et « les aider à apprendre ».

\section{Une implication dans les activités pédagogiques}

Si l'enquête par questionnaire montrait déjà que, davantage qu'une aide matérielle dans la vie quotidienne, la fonction essentielle des AVS consiste à apporter une aide dans les activités scolaires, la description qu'ils font de leurs pratiques met en évidence sa composante pédagogique. Leurs interventions pour faciliter la compréhension et la réalisation du travail par les élèves ne se limitent pas à une simple répétition des consignes. II s'agit de les reformuler différemment, de donner des explications supplémentaires sur le contenu du cours, parfois des pistes 
de travail pour aborder les exercices, voire de simplifier le travail demandé ou de concevoir des activités accessibles aux élèves suivis. Une " contradiction » est soulignée entre leur fonction officielle, telle qu'elle leur a été présentée en formation, et leurs interventions effectives sur le terrain. Ils ont retenu qu'ils n'ont pas à intervenir sur le plan de l'enseignement. Cependant, à leur avis, l'aide apportée aux élèves dans les tâches scolaires constitue bien " un accompagnement pédagogique ", bien qu'aucune compétence, dans ce domaine, ne leur soit reconnue. Ces résultats rejoignent les conclusions de différents travaux qui mettent en avant la complexité de la fonction d'AVS (Fédération nationale des associations au service des élèves présentant une situation de handicap, 2002) et le fait qu'elle met en jeu des compétences proches de celles des métiers de l'éducation et de l'enseignement (Bordeau \& Bourget, 2009 ; Philip, 2009).

Cette mise en jeu de compétences pédagogiques n'induit pas pour autant de confusion des rôles entre AVS et enseignants. Ces derniers sont reconnus comme les responsables du fonctionnement pédagogique de la classe et le travail d'accompagnement des AVS s'effectue sous leur contrôle : « Surtout, ne pas remplacer le professeur, ne pas intervenir à sa place, sinon, on sort du cadre de notre fonction. " Les AVS se placent en position de second, disant intervenir en fonction des directives des enseignants et, dans leurs initiatives, en référer à eux : « Je m'applique à bien être le relais de ce que demande l'enseignante. " Ils ont conscience de ne pas disposer des mêmes compétences que les enseignants : " Je pourrais faire des erreurs importantes en essayant d'expliquer les choses de façon incorrecte. " S'il arrive parfois qu'ils soient conduits à suppléer l'enseignant (par exemple, en prenant en charge un groupe d'élèves lors de son absence), ils n'assurent cette tâche, qu'ils savent dépasser leurs attributions, que par nécessité, pour ne pas abandonner les élèves et au prix d'un net malaise. Dans le discours des AVS, la délimitation des sphères d'intervention paraît ainsi s'établir clairement, leur rôle ne pouvant être assimilé à celui des enseignants, même si dans la réalité, certains faits contreviennent à cette répartition du travail.

\section{UN REGARD CRITIQUE SUR LA FORMATION}

Pour acquérir les savoir-faire et les connaissances qu'ils estiment nécessaires à l'exercice de leur fonc- tion, les AVS disposent, d'après les textes officiels qui les régissent, d'une formation à l'emploi de 60 heures obligatoires. Ce n'est pourtant pas ce qu'ils mentionnent le plus souvent en premier lieu. Ils mettent plutôt en avant l'apprentissage " sur le terrain ", par la pratique avec les enfants et les contacts avec leurs partenaires, essentiellement les enseignants et des professionnels spécialisés qui suivent les enfants. Ils accordent moins d'importance à leurs échanges avec les parents comme moyen de développer leurs compétences professionnelles. Par contre, ils s'appuient sur leurs expériences antérieures dans le domaine éducatif et mobilisent parfois des connaissances acquises au cours de leurs études. Ils ont également recours à des recherches personnelles de documentation, notamment sur Internet. De manière générale, leurs appréciations sur la formation dispensée sont plutôt négatives, bien qu'ils ne contestent pas son intérêt. Elle apporte, en effet, des informations qu'ils estiment utiles pour assurer leur fonction, principalement sur les différents types de handicap ainsi que sur leur rôle d'AVS et, dans une moindre mesure, sur la législation, les structures et dispositifs existants.

\section{Une formation trop tardive}

Ces informations leur sont cependant données de façon trop tardive. Ils ont l'impression d'être " parachutés » sur le terrain. Leur discours témoigne de la violence avec laquelle ils ont vécu ce premier contact : "On jette les gens, allez, débrouillezvous! "; «On est jeté dans la fosse aux lions ». Une des principales difficultés rencontrées par les AVS est effectivement le fait d'arriver dans un établissement sans rien connaître de son fonctionnement ni des difficultés des enfants : "La prise de fonction a été un peu brutale [...] j'étais complètement perdue. "Sans ces informations préalables, ils en sont réduits à une totale improvisation sur le terrain. Ils réclament donc avec force un minimum de préparation avant de prendre leur poste.

\section{Une formation trop théorique}

La formation reçue est aussi très souvent décrite comme trop " théorique ", c'est-à-dire trop générale, trop éloignée des pratiques et déconnectée de la réalité de leur travail. Elle ne leur permet pas de savoir comment intervenir auprès des enfants : "C'était intéressant dans le sens où on apprend tous les troubles qui existent, mais après, sur le terrain, 
qu'est-ce qu'on en fait? "Par contre, toutes les occasions qui sont fournies, au cours de la formation, d'aborder concrètement les pratiques sont unanimement appréciées. En particulier, les moments d'échanges entre AVS sont jugés enrichissants. Ils ont un effet rassurant et le partage d'expériences peut suggérer des pistes sur la façon de travailler avec les élèves : "Ça nous a permis de voir qu'on n'était pas seul à se sentir seul [...] et puis de faire un peu le point sur nos pratiques et de voir ce qui se passait ailleurs. " De même, sont jugées bénéfiques, car " c'est du pratique » : les interventions de professionnels et de parents faisant part de leur expérience avec des enfants handicapés, le témoignage de personnes handicapées elles-mêmes (par exemple autistes), des rencontres entre enseignants spécialisés des dispositifs collectifs (classes CLIS ou unités spécialisés UPI) et AVS, un travail mené sur l'utilisation d'un " cahier de bord ", sur lequel noter les réactions des enfants, leur évolution, etc. Ceux qui ont bénéficié de la visite d'un établissement spécialisé estiment que cette expérience est profitable. Elle est l'occasion pour eux d'une découverte de la réalité du handicap et le moyen de voir comment des enseignants spécialisés travaillent avec ces enfants. II apparaît ainsi que ce qui préoccupe au premier plan les AVS, ce sont les répercussions du handicap sur le fonctionnement intellectuel des enfants et leur comportement social, et surtout les pratiques professionnelles qu'il est possible d'envisager avec eux.

\section{Une formation trop limitée}

Un autre reproche concerne le temps de formation qu'ils estiment trop court ${ }^{10}$. II est à noter à cet égard que les AVS qui en sont le plus satisfaits sont ceux qui ont pu suivre l'ensemble de la formation proposée, à la fois des heures obligatoires d'adaptation à l'emploi, organisées par l'inspection académique, et des modules complémentaires facultatifs. Ces modules, prévus dans les textes et pouvant aller jusqu'à 120 heures, visent un approfondissement des connaissances abordées au cours de la formation obligatoire ${ }^{11}$. Cependant les conditions dans lesquelles ces modules se déroulent n'incitent pas toujours les AVS à y participer car ils ne sont par exemple pas compris dans les heures de service. Les AVS interrogés souhaitent généralement avoir la possibilité de poursuivre leur formation pour approfondir les premiers acquis ou les compléter sur des points particuliers en rapport avec leurs expériences. De même, ils apprécieraient que des rencontres entre AVS soient renouvelées régulièrement. Ils expriment éga- lement le besoin d'un accompagnement après la formation, qui leur apporterait une aide face aux problèmes auxquels ils sont confrontés sur le terrain (par exemple sous la forme de groupes réguliers d'analyse de pratiques, comme le suggère une AVS).

\section{L'IMPORTANCE ACCORDÉE AUX RELATIONS AVEC LES ENSEIGNANTS DES CLASSES D'ACCUEIL}

De par leurs fonctions, les AVS sont amenés à travailler en premier lieu avec les enseignants des classes dans lesquelles se trouvent les élèves qu'ils sont chargés de suivre (classes ordinaires pour les AVSi, dispositifs collectifs des CLIS ou des UPI pour les AVSco). Ils estiment qu'ils peuvent tirer profit de ces contacts pour améliorer leurs compétences. Les échanges avec les enseignants permettent aux AVS de comprendre les objectifs et les méthodes pédagogiques développées dans la classe. Ils sont aussi l'occasion de s'accorder sur des modalités d'intervention, voire de rechercher ensemble des solutions pour répondre aux besoins d'élèves en difficulté. Cependant la qualité de ces apports dépend du mode d'interactions qui s'instaure avec ces partenaires. La situation sur ce plan s'avère contrastée selon qu'ils sont AVSi ou AVSco.

Les AVSco s'estiment bien accueillis par les enseignants spécialisés de la CLIS ou de I'UPI dans laquelle ils sont affectés, ceux-ci ayant déjà des idées sur la façon de tirer parti de leur présence pour les aider dans la gestion du groupe d'élèves. Ils font état d'une collaboration étroite avec l'enseignant de la classe : "On fonctionne vraiment bien ensemble, on discute beaucoup ", certains allant jusqu'à parler de rapports de "complicité ». Le fait de partager l'encadrement d'un même groupe d'élèves facilite les échanges qui ont lieu en fonction des besoins, au cours des activités, mais aussi dans les interclasses ou après la classe. Les AVSco sont associés à tout ce qui concerne le fonctionnement de la classe. Ils se considèrent comme des " assistants " de l'enseignant: " Je suis là pour aider le maître à faire ce qu'il avait prévu. " Dans le cadre de cette collaboration, les enseignants spécialisés assurent un encadrement permanent des AVSco avec lesquels ils travaillent. Au départ, ce sont souvent eux qui les introduisent dans leur fonction, en leur donnant de premières indications sur leur rôle. En explicitant leurs attentes, ils donnent des repères aux AVS sur la façon d'intervenir 
auprès des élèves : “Au début, je me posais beaucoup de questions [...] il m'a un peu expliqué, heureusement qu'il était là. » En tant qu'enseignant spécialisé, ils sont également en mesure d'apporter des explications sur les difficultés des enfants, de donner des pistes de travail : " Je pourrais dire qu'elle est mon guide. "Ils sont un recours sécurisant en cas de difficulté : «Dès que j'ai un souci, je vois avec elle. »

En général, les AVSco interrogés rendent compte d'une expérience très positive. Ils évoquent des modalités de travail définies en concertation avec l'enseignant spécialisé et parlent également de sa professionnalité ("Elle est très compétente sur tous les petits trucs qu'on peut trouver pour faire comprendre les enfants »), des rapports de confiance («Elle m'a dit: "Tu essaies, si ça ne marche pas, tu peux tenter autre chose, et si tu as besoin, on voit ensemble" "). II apparaît que, dans ce climat de reconnaissance mutuelle, les auxiliaires se sentent autorisés à faire des propositions dans le cadre défini par l'enseignant: "Souvent, les choses que je dis, l'enseignante les prend en compte [...] c'est vraiment un échange que j'espère constructif. "Dans ces conditions, la collaboration avec les enseignants spécialisés a un rôle éminemment formateur. Les AVSco s'approprient leurs savoir-faire, au point que certains se disent en mesure de les transmettre à d'autres enseignants, notamment lorsqu'ils accompagnent un élève dans une autre classe : " Je donne mon avis parce que je sais comment ça se passe avec monsieur B. "Une seule AVSco évoque des difficultés, rencontrées l'année précédente, avec une enseignante qui a abandonné sa fonction en cours d'année et avec les remplaçants qui se sont ensuite succédé. Ceci nous conduit à faire l'hypothèse que ce cas de figure est plutôt marginal. De façon générale, il apparaît que le fait d'être encadré par un enseignant spécialisé avec lequel ils ont des échanges permanents place les AVSco dans de meilleures conditions que les AVSi pour acquérir les moyens d'accomplir leur mission d'accompagnement de façon satisfaisante.

En effet des AVSi relatent des relations difficiles avec certains enseignants. Ils se disent mal acceptés et pensent que cela provient de la gêne ressentie par les enseignants lorsqu'un autre adulte est présent dans la classe : "Un adulte peut toujours porter un regard et avoir un avis différent. [...] on peut même avoir des critiques et c'est difficile à accepter pour un enseignant, je le comprends. " Ils l'expliquent également par des réticences envers l'accueil d'élèves handicapés, par un manque de considération à leur égard ou des réserves concernant la fonction d'AVS.
Dans ce cas, tout juste tolérés dans la classe, ils n'ont que des échanges très limités avec les enseignants et c'est à eux seuls qu'incombe la responsabilité d'adapter le travail de la classe pour l'enfant qu'ils accompagnent.

La méconnaissance de leur rôle par les enseignants conduit à des malentendus, comme par exemple la crainte de voir leur responsabilité empiétée : "Elle ne voulait pas [que j'intervienne] parce que, en gros, je lui piquais son travail » ou, au contraire, l'attente de compétences spécialisées de la part de l'AVS : "Les instituteurs, ils nous attendent comme une aide, alors, si on arrive et qu'on n'est pas une aide..." Cette méconnaissance contribue, de leur point de vue, à la méfiance ou au rejet dont ils sont parfois l'objet et ne facilite pas l'établissement de relations de collaboration. Ces difficultés peuvent être atténuées par le fait que les AVSi, en suivant plusieurs élèves, sont amenés à travailler avec d'autres enseignants et peuvent avoir ainsi l'occasion de développer d'autres relations plus satisfaisantes. Ils trouvent également des possibilités d'échanges avec d'autres partenaires (professionnels spécialisés, documentalistes...). Certains, par leurs expériences professionnelles antérieures, ont acquis des compétences relationnelles qui leur permettent d'affronter ces difficultés : "Moi, j'ai un peu d'âge, il y a des choses qui ne m'intimident pas. "Ils s'efforcent d'instaurer progressivement un climat d'échanges : "C'est moi qui lui parle spontanément [de l'enfant], mais elle, elle ne me demande pas [...] En fait, on n'est pas de réelles partenaires. [...] Mais ce sont des choses qui se construisent. Et effectivement, maintenant, on arrive à la fin de l'année et je me rends compte que petit à petit, les relations ont évolué. Mais c'est vrai que c'est long, c'est tout un travail. »

Par contre, même quand AVSi et enseignants partagent la même préoccupation de rechercher des situations adaptées aux enfants, ils peuvent se retrouver, l'un comme l'autre, démunis devant certains enfants qui posent problème : “C'est un peu dangereux parce que, parfois, je pense qu'on peut faire des erreurs. Parce qu'on est un peu tout seul, même s'il y a l'enseignant, mais l'enseignant n'en sait pas plus que nous, il est habitué à travailler avec des enfants "classiques". " II apparaît ainsi que les AVSi peuvent se trouver en difficulté, tant pour aider les enfants que pour développer des compétences relatives à cette fonction, du fait du manque de préparation des enseignants à accueillir des élèves en situation de handicap et à travailler avec des auxiliaires de vie scolaire. La question de la réparti- 
tion des rôles avec les enseignants au sein de la classe n'est pas spécifique aux AVS, elle se pose pour tout intervenant extérieur. Elle paraît cependant revêtir un caractère particulier du fait de l'ambiguïté de leur position. En effet, alors qu'ils ne sont pas censés disposer de compétences spécifiques, au même titre que les assistants d'éducation, leur affectation auprès d'enfants à titre individuel (en ce qui concerne les AVSi) peut les conduire à assurer la responsabilité de l'adaptation du travail pour des élèves handicapés.

\section{LE BESOIN D'ÉCHANGES AVEC D'AUTRES PARTENAIRES}

\section{Le soutien d'un travail en équipe}

Au-delà des enseignants impliqués en premier lieu dans l'accueil des élèves handicapés, des relations peuvent également se développer avec d'autres enseignants de l'établissement. Lorsqu'ils accompagnent des élèves de CLIS ou d'UPI intégrés ponctuellement en classe ordinaire, les AVSco se heurtent parfois aux mêmes écueils que les AVSi avec certains enseignants des classes d'accueil : présence de l'AVS en classe mal supportée, voire refusée, faible engagement dans l'accueil de ces élèves.

Par contre, qu'ils soient AVSco ou AVSi, quand les enseignants se montrent favorables à l'accueil d'élèves en situation de handicap, les contacts s'établissent facilement et peuvent être source d'apprentissage : «J'ai pêché ce que je voyais autour de moi [...], la façon dont ils expliquaient, je piquais des petites idées à droite et à gauche. " De plus, quand un travail d'équipe existe, l'AVS est tout naturellement inscrit dans ce réseau d'échanges et bénéficie du climat de réflexion collective qui règne dans l'école : “On peut oser dire qu'on n'y arrive pas et demander de l'aide, des démarches communes sont faites. "Plusieurs AVS soulignent également que l'attitude du chef d'établissement ou du directeur d'école a une incidence importante sur l'accueil d'élèves handicapés dans l'établissement. Par son rôle d'animation pédagogique, il peut influer sur le développement de relations de coopération au sein de l'école comme avec des partenaires extérieurs : " II était au cœur du projet, il voulait vraiment accueillir des enfants handicapés, et il assiste à nos réunions, il se tient au courant. "Quand il s'implique dans la résolution des problèmes rencontrés (par exemple par une écoute attentive ou en suscitant une réflexion en équipe), les échanges avec lui, de même qu'avec les enseignants, constituent un soutien et peuvent contribuer à la construction de savoir-faire pour les AVS.

\section{Les professionnels spécialisés : des personnes ressources}

Les professionnels spécialisés (de l'Éducation nationale ou du secteur médico-social ou sanitaire) sont généralement considérés par les AVS comme des personnes dotées d'une expertise et sur lesquelles ils peuvent compter. Quand des contacts entre eux existent, la mise en commun des observations réalisées dans des contextes différents (école et lieu de soins) permet aux AVS d'accéder à une meilleure compréhension de leur comportement et d'ajuster des attitudes éducatives : «Ça nous permet d'avoir un regard différent [...], de savoir ce qu'on peut attendre [de l'enfant] ou pas. "Les échanges débouchent parfois sur une utilisation des pratiques employées en rééducation (par exemple reprise en classe de documents établis par l'orthophoniste). Les interventions d'enseignants spécialisés itinérants rattachés au "pôle ressources " de l'inspection académique du département sont particulièrement appréciées par les AVSi qui en ont bénéficié : «Elle m'a donné plein de pistes. " Du fait de leur connaissance de l'école et de la pratique avec les enfants dans ce contexte, ces enseignants leur donnent des indications concrètes qu'ils jugent particulièrement adaptées à leurs attentes. Mais leurs interventions, une à trois fois au maximum dans l'année, ne peuvent suffire à assurer un encadrement tel que celui que les AVSco trouvent quotidiennement auprès des enseignants spécialisés de CLIS ou d'UPI.

Lorsque les rencontres avec les professionnels spécialisés sont fréquentes, elles peuvent donner lieu à une collaboration plus étroite avec les AVS, comme cela se produit avec des enseignants de classes ordinaires (Belmont \& Vérillon, 2004). II est fait état d'expériences très positives avec ces professionnels lorsqu'ils interviennent régulièrement à l'école. Ainsi la possibilité d'assister à la séance hebdomadaire de kinésithérapie dans l'école apporte à une AVS une meilleure connaissance de l'évolution des capacités motrices d'un élève. Elle est aussi l'occasion d'acquérir de nouveaux savoir-faire (comment le porter, l'aider dans ses mouvements...). La présence de la psychologue d'un SESSAD12 dans une UPI, une journée par semaine, permet de nombreux échanges sur le travail des élèves ou sur leurs difficultés. 
De façon générale, les contacts avec les professionnels spécialisés sont estimés insuffisants. Les relations peuvent aussi être inexistantes ou encore jugées superficielles, sans réelle volonté de collaboration, parfois même avec une équipe de soins qui est installée dans l'établissement scolaire. Des AVS évoquent ce qu'ils ont perçu comme un « conflit entre l'équipe enseignante et l'équipe de santé, [...] entre les deux ministères ", ainsi que les désaccords qui peuvent subsister : "On se heurte parfois à des problèmes de compréhension, parce qu'on n'a pas la même façon de travailler. " Ainsi il ne suffit pas que les rencontres soient matériellement possibles, encore faut-il que le développement de relations de collaboration avec l'école fasse partie des conceptions de travail des professionnels spécialisés. Ces professionnels apparaissent néanmoins aux yeux des AVS comme possédant des compétences spécifiques. Les AVS recherchent alors auprès d'eux des informations, des savoirs, des pistes de travail dont ils estiment avoir besoin pour parvenir à une meilleure maîtrise de leur travail. S'ils n'ont pas toujours une connaissance très précise des rôles respectifs des différents professionnels avec lesquels ils sont en contact, ils leur reconnaissent cependant un domaine d'intervention propre, qui ne recouvre pas celui des AVS. Cela est particulièrement net lorsqu'il s'agit de professionnels du secteur médical ou paramédical. Par contre, la frontière entre les tâches des AVS et celles des éducateurs spécialisés semble plus floue. Une AVS dit ainsi avoir des difficultés à se situer quand l'éducatrice spécialisée intervient dans la classe, ce qui nécessiterait un ajustement de leurs modalités de travail. En l'occurrence, elle règle le problème en s'effaçant devant la spécialisation de sa partenaire, "parce que c'est quand même l'éducatrice spécialisée ", et en adoptant alors une posture d'observatrice. Cette proximité des tâches conduit un des AVS interrogés à penser que le travail d'AVS nécessite des compétences proches de celles d'éducateur spécialisé et donc une formation comparable : «Savoir quels sont les comportements d'un adolescent ou d'un enfant, comment on peut répondre à leurs attentes [...], connaître leur handicap, leurs besoins, comment intervenir lorsqu'ils sont en difficulté. " Tout en revendiquant la reconnaissance de la fonction d'AVS comme métier, il estime qu'il serait important de favoriser la présence d'éducateurs spécialisés au sein de l'Éducation nationale. Une double question est alors posée : d'une part, celle de l'opportunité ou non d'instaurer un nouveau corps de métier d'AVS ; d'autre part, celle de la nécessité de spéci- fier les fonctions remplies par les différents professionnels susceptibles d'apporter un appui à la scolarisation d'élèves en situation de handicap dans les établissements ordinaires.

\section{CONCLUSION}

L'instauration des auxiliaires de vie scolaire pour assurer l'accompagnement des enfants handicapés au sein de l'école prend place dans les processus de division du travail éducatif. Ces phénomènes, qui concernent aussi les personnels regroupés sous le titre d'assistants d'éducation, acquièrent cependant ici la particularité de ne plus se situer dans le prolongement de la spécialisation d'enseignants dédiés aux « inadaptations » puis aux « handicaps ", mais de viser des nouveaux venus dont les activités sont définies comme provisoires, comme fournissant seulement un appui à la scolarisation des enfants handicapés. Bien que revendiqués fortement par les parents et par diverses associations, ce sont des personnels seconds par rapport aux professeurs en place et qui se situent surtout dans le cadre d'une mission précaire. Comme l'étaient les aides éducateurs, ils sont « en transit au sein de l'Éducation nationale " (Cadet, Diederichs-Diop, Fournié et al., 2000). Cette nouvelle division du travail, instaurée au nom de l'accompagnement nécessaire d'enfants en situation de handicap, risque de se traduire par des difficultés à assurer la qualité éducative qui en est attendue. L'objectif de cet article consistait précisément à examiner les relations entre le statut accordé aux AVS et l'exercice concret de leur fonction d'accompagnement. Partant de leurs témoignages, nos analyses nous ont conduits à mieux cerner leurs positions subjectives à la fois sur leurs conditions de travail et sur leur activité, ainsi qu'à avancer dans la compréhension de leur revendication de nouvelles conditions d'emploi.

De fait, les 34 AVS interrogés dans le département de Seine-Saint-Denis considèrent que le statut conféré à leur emploi n'est pas à la hauteur de la fonction attendue. C'est, disent-ils, un travail exigeant, difficile, qui suppose des compétences diverses et un fort engagement personnel. Ils se réfèrent ainsi à divers registres de professionnalité : choix éthique par rapport au handicap, compétences relationnelles, qualités humaines et capacités d'adaptation, mais aussi compétences d'ordre pédagogique, dès qu'il est question d'apprentis- 
sages scolaires. En termes de professionnalisation, il paraît déterminant aux AVS étudiés que leur activité, dont ils parlent comme d' " un vrai métier », fasse l'objet d'une reconnaissance sociale. En ce sens, leurs propos font écho à des travaux qui montrent que "plus les acteurs s'appuient sur un métier, plus leur expérience de travail est sereine ", l'idée de métier impliquant "que le travail soit objectivable et que le professionnel puisse dire : ceci est mon œuvre, ceci est le résultat de mon activité » (Dubet, 2002, p. 393). Leurs revendications en faveur d'un métier reconnu sont aussi intimement liées à une demande de formation ajustée à leurs besoins, qui se situent sur trois plans:

- une préparation avant la prise de fonction ;

- un contenu de formation davantage orienté vers les pratiques avec les élèves ;

- un accompagnement dans leur pratique professionnelle pour approfondir leurs acquis, en regard de leur expérience de terrain.

Des dispositifs de formation ont été expérimentés dans plusieurs départements pour articuler apports de connaissances et réflexion sur la pratique (Philip, 2009 ; Cagnioncle, 2009 ; Toullec-Théry, Dujardin, Nédélec-Trohel et al., 2009), mais ils nécessitent du temps et un échelonnement dans l'année difficiles à mettre en œuvre dans le cadre des 60 heures obligatoires d'« adaptation à l'emploi ». Peu d'AVS bénéficient des modules complémentaires facultatifs. Des initiatives ministérielles tentent de prendre en compte cette préoccupation d'une meilleure formation des AVS. Elles annoncent notamment « un accompagnement individualisé pendant l'exercice de l'activité » (MEN, 2008). Mais celui-ci est envisagé en fin d'année, comme " une évaluation de chaque agent ainsi qu'une analyse de ses besoins de formation » (MEN, 2008), en vue d'un métier ultérieur. II ne vise donc pas une aide au développement de leur pratique professionnelle en tant qu'AVS.

En l'état actuel de leur statut, le dispositif d'accompagnement des élèves handicapés repose essentiellement sur la motivation des AVS et leur capacité à assurer une " autoformation ", à partir de leurs propres ressources : cursus d'études, expériences antérieures, démarches personnelles ${ }^{13}$. Leur reconnaissance se fonde ainsi sur une individualisation des compétences, au lieu de se référer à des pratiques d'accompagnement institutionnellement établies. Pour développer leurs compétences, les AVS sont non seulement peu aidés par la formation, mais ils souffrent de plus de faiblesses persistantes dans la mise en œuvre de la scolarisation des élèves handicapés :

- le manque de formation, dans ce domaine, des enseignants qui exercent dans les classes ordinaires ;

- l'existence encore peu courante du travail en équipe entre enseignants ;

- la faible collaboration entre l'établissement scolaire et le milieu médico-social.

En effet le développement des relations avec leurs partenaires (enseignants et professionnels spécialisés) se révèle primordial pour permettre aux AVS de comprendre et maîtriser leur fonction d'accompagnement. C'est grâce à ces échanges qu'ils parviennent à trouver leur place par rapport aux autres acteurs et à déterminer progressivement leur champ d'action. C'est auprès de ces partenaires qu'ils cherchent à puiser ce qui pourrait leur permettre de progresser dans leur pratique. L'efficience de tels échanges dépend cependant de leur inscription dans une démarche de collaboration, prenant appui sur les compétences de chacun, pour rechercher des moyens d'actions adaptés à partir d'un partage d'observations. En cela, l'expérience des AVS renvoie à ce que Serge Ebersold (2003) nomme un « système équitable de coopération ". En réalité, les relations de coopération sont plus favorables pour les AVS dits AVSco, qui se situent dans des dispositifs collectifs (classes ou unités), que pour les AVSi qui ont la tâche de s'occuper individuellement d'enfants handicapés et qui se trouvent le plus souvent dispersés entre plusieurs classes. On voit bien le bénéfice que constitue pour les premiers non seulement la stabilité d'un même lieu de travail, mais aussi l'apport professionnel de la part d'enseignants spécialisés, qui s'appuient à la fois sur une expérience du milieu scolaire et sur le travail avec des enfants à besoins éducatifs particuliers. On peut s'interroger sur le choix fait en France d'affecter ces enseignants spécialisés en majorité dans les dispositifs collectifs d'intégration ou dans des établissements spécialisés, alors que leur apport pourrait également bénéficier à des équipes, enseignants et AVS, exerçant en classe ordinaire.

Pour développer le partenariat, la préparation de l'ensemble des enseignants de classe ordinaire à l'accueil d'élèves handicapés et au travail en collaboration avec des AVS apparaît comme une nécessité. Les réflexions actuelles sur la formation des enseignants mettent l'accent autant sur le développement de leurs capacités à établir des coopérations interprofessionnelles que sur la prise en compte de la 
diversité des besoins des élèves (De Anna, 2009 ; Plaisance, 2009a). Pour que les professionnels du milieu médico-social puissent aussi jouer le rôle de personnes ressources pour les AVS comme pour les enseignants, il semble indispensable que ce rôle soit effectivement reconnu comme faisant partie de leur mission et qu'il s'inscrive dans leurs orientations de travail. Cela suppose une évolution des cultures professionnelles vers la construction de cultures partagées, notamment par l'instauration de modules communs de formation initiale et continue (Chauvière \& Plaisance, 2008 ; Plaisance, 2009b).

Brigitte Belmont CERLIS, université Paris-Descartes

Éric Plaisance eric.plaisance@paris5.sorbonne.fr CERLIS, université Paris-Descartes

Aliette Vérillon

UMR P3 ADEF, Institut français de l'Éducation, École normale supérieure de Lyon

\section{NOTES}

1 Loi n 2005-102 du 11 février 2005 pour l'égalité des droits et des chances, la participation et la citoyenneté des personnes handicapées, disponible sur Internet à l'adresse : <http://www. legifrance.gouv.fr/affichTexte.do?cidTexte=JORFTEXT000000809647> (consulté le 26 janvier 2011).

2 L'accompagnement par les AVS consiste à « optimiser l'autonomie de l'enfant dans ses apprentissages, faciliter sa participation aux activités collectives et aux relations interindividuelles, assurer son installation dans des conditions optimales de sécuassurer son installation dans des conditions optimales de sécu-
rité et de confort ", d'après la loi $n^{\circ} 2003-400$ du 30 avril 2003 rité et de confort ", d'après la loi $n^{\circ} 2003-400$ du 30 avril 2003
relative aux assistants d'éducation, disponible sur Internet relative aux assistants d'éducation, disponible sur Internet
à l'adresse : <http://www.legifrance.gouv.fr/affichTexte.do?cidTe $x$ te $=$ JORFTEXT000000602740\&dateTexte $=>$ (consulté le 26 janvier 2011).

3 «En mars 2009, 9728 emplois d'assistants d'éducation AVSi (individuels) et 2083 emplois d'assistants d'éducation AVSco (collectifs) étaient occupés. Pour compléter l'intervention des AVSi, des personnels ont été recrutés sur des contrats d'accompagnement dans l'emploi (CAE) ou sur des contrats d'avenir (CAV), soit plus de 12679 personnes (7 454 équivalents temps plein). À la rentrée 2010, 57067 élèves font l'objet d'un accompagnement individuel. On dénombre 21800 équivalents temps plein AVSi et 2166 équivalents temps plein AVSco " (MEN, 2010).

4 Dans la loi $n^{\circ}$ 2005-102 du 11 février 2005, c'est sous le titre " accessibilité " que sont précisées les modalités de scolarisation.

5 C'est le cas, par exemple, des dispositifs intitulés « services d'accompagnement à la vie sociale " et " services d'accompagnement médico-social pour adultes handicapés ".

6 Selon ces mêmes auteurs, " un emploi est de qualité si les rémunérations associées permettent de dépasser le seuil de pauvreté, si la stabilité de l'emploi et la sécurité économique sont assurées, si les conditions de travail ne sont pas préjudiciables à la santé et enfin si la reconnaissance juridique et symbolique associée à l'emploi est suffisante " (Devetter, Jany-Catrice \& Ribault, 2009, p. 62)
7 Dans l'article considéré, l'auteur analyse la garde des enfants des autres à domicile, en mettant l'accent sur le rôle des pouvoirs publics dans le processus de professionnalisation des assistantes maternelles. Ce faisant, il considère ce métier comme un métier féminin, écartelé dit-il entre «le travail d'éveil de la puériculture de la crèche et celui de l'institutrice d'école maternelle » (Aballea, 2005).

8 II ne nous a pas semblé pertinent d'interroger des EVS, car l'année 2005-2006 était la première année d'installation de ces emplois.

9 Cette référence à la " bonne » ou « juste " distance est aussi fortement présente dans les analyses de l'accompagnement des personnes handicapées en général (voir l'ouvrage de Stiker, Puig \& Huet, 2009, p. 46).

10 Sur l'ensemble de la population d'AVS du département, seule une minorité d'entre eux a bénéficié de plus d'une semaine de formation (49\% des AVSi et $30 \%$ des AVSco).

11 Ils sont assurés, dans le département considéré, par le Centre national d'éducation et de formation pour l'enfance inadaptée (CNEFEI), actuellement INS HEA (Institut national supérieur de formation et de recherche pour l'éducation des jeunes handicapés et les enseignements adaptés).

12 SESSAD : Service d'éducation spécialisée et d'aide à domicile.

13 L'orientation officielle actuelle est d'augmenter le nombre d'EVS au détriment des AVS. Les problèmes évoqués par les AVS risquent d'être beaucoup plus difficiles à surmonter par ces personnels qui disposent, pour remplir une fonction comparable, de moins de ressources en termes de formation initiale et d'expérience professionnelle. En outre, ils sont encore aujourd'hui peu nombreux à recevoir une formation. Environ $50 \%$ des EVS n'ont reçu aucune formation en fin d'année scolaire 2007-2008, ce qui va dans le sens de nos résultats précédents, ainsi que de l'enquête de l'Union nationale pour l'avenir de l'inclusion scolaire, sociale et éducative (2008)

\section{BIBLIOGRAPHIE}

ABALLEA F. (2005). " La professionnalisation inachevée des assistantes maternelles ». Recherches et prévisions, $\mathrm{n}^{\circ} 80$, p. 55-65.

BELMONT B. \& VÉRILLON A. (2004). « Relier les territoires par la collaboration des acteurs ». In D. Poizat, Éducation et handicap. D'une pensée territoire à une pensée monde. Toulouse : Érès, p. 57-66.
BELMONT B., PLAISANCE É. \& VÉRILLON A. (2006). "Accompagnement et intégration scolaire. Politique, pratiques et acteurs ». Contraste. Enfance et handicap, $n^{\circ} 24$, p. 247-266.

BELMONT B., PLAISANCE É. \& VÉRILLON A. (2009). « Les auxiliaires à l'intégration scolaire des enfants en situation de handicap. Conditions de travail et développe- 
ment de compétences professionnelles ». Alter. Revue européenne de recherche sur le handicap, vol. $3, n^{\circ} 4$, p. 320-339.

BLANC P. (2007). Rapport d'information $n^{\circ} 359$ fait au nom de la commission des Affaires sociales sur l'application de la loi $n^{\circ}$ 2005-102 du 11 février 2005 pour l'égalité des droits et des chances, la participation et la citoyenneté des personnes handicapées. Paris : Sénat. Disponible sur Internet à l'adresse : <http://www.senat. $\mathrm{fr} / \mathrm{rap} / \mathrm{rO6}-359 / \mathrm{r06}-359$ mono.html $>$ (consulté le 26 janvier 2011).

BORDEAU M. \& BOURGET G. (2009). « Le personnel accompagnant scolaire, une approche sociohistorique ". La nouvelle revue de l'adaptation et de la scolarisation, $\mathrm{n}^{\circ} 45$, p. 55-69.

BOURDONCLE R. (2000). « Autour des mots. Professionnalisation, formes et dispositifs ». Recherche et formation, $\mathrm{n}^{\circ} 35, \mathrm{p} .117-132$.

BOYÉ V. (1998). Les auxiliaires d'intégration scolaire dans le Bas-Rhin. Formation, fonction, population prise en charge. Strasbourg : Centre régional pour l'étude et l'action en faveur des personnes inadaptées. Disponible sur Internet à l'adresse : <http://www. creaialsace.org/IMG/pdf/Etudes/IS01.pdf> (consulté le 26 janvier 2011).

CADET J.-P. (2008). «Qu'est-ce que la professionnalisation? » In J.-J. Paul \& J. Rose (dir.), Les relations formationemploi en 55 questions. Paris : Dunod, p. 339-344.

CADET J.-P., DIEDERICHS-DIOP L., FOURNIÉ D. \& GUITTON C. (2000). "Intégration, professionnalisation, mobilité : la valse à trois temps des aides éducateurs ». Bref, $\mathrm{n}^{\circ} 167$, p. 1-4.

CAGNIONCLE M. (2009). « Une formation pour les auxiliaires de vie scolaire ". La nouvelle revue de l'adaptation et de la scolarisation, $\mathrm{n}^{\circ} 45$, p. 110-116.

CASTEL R. (2009). La montée des incertitudes. Travail, protections, statut de l'individu. Paris : Éd. du Seuil.

CHAUVIÈRE M. \& PLAISANCE É. (2008). "Les conditions d'une culture partagée ». Reliance, $\mathrm{n}^{\circ} 27$, p. 31-44.

COMMISSION NATIONALE CONSULTATIVE DES DROITS DE L'HOMME (2008). Avis sur la scolarisation des enfants handicapés. Paris : Commission nationale consultative des droits de l'Homme. Disponible sur Internet à l'adresse : <http://www.cncdh.fr/article. php3?id_article=584> (consulté le 26 janvier 2011).

DE ANNA L. (2009). «Formation en Italie des enseignants accueillant des enfants handicapés ". Recherche et formation, $\mathrm{n}^{\circ} 61$, p. 55-70.

DEVETTER F.-X., JANY-CATRICE F. \& RIBAULT T. (2009). Les services à la personne. Paris : La Découverte.

DUBET F. (2002). Le déclin de l'institution. Paris : Éd. du Seuil.

EBERSOLD S. (2003). "Intégration scolaire, dynamiques de scolarisation et logiques d'accompagnement ». Handicap, $\mathrm{n}^{\circ}$ 99, p. 17-32.

EBERSOLD S. (2009). «Auxiliaires de vie scolaire. Légitimité professionnelle et système équitable de coopération ". La nouvelle revue de l'adaptation et de la scolarisation, $\mathrm{n}^{\circ} 45$, p. $118-149$.
FÉDÉRATION NATIONALE DES ASSOCIATIONS AU SERVICE DES ÉLÈVES PRÉSENTANT UNE SITUATION DE HANDICAP (2002). Référentiel d'emploi de la fonction d'auxiliaire de vie scolaire. Disponible sur Internet à l'adresse : <http://mimibaby.free.fr/ referentiel\%20avs\%20fnaseph.htm> (consulté le 26 janvier 2011).

GEOFFROY G. (2006). Réussir la scolarisation des enfants handicapés. Rapport au ministère de l'Éducation nationale, de l'Enseignement supérieur et de la Recherche, au ministère de la Santé et des Solidarités et au ministère de la Sécurité sociale, des Personnes âgées, des Personnes handicapées et de la Famille. Disponible sur Internet à l'adresse : < http://www. ladocumentationfrancaise.fr/rapports-publics/064000842/ index.shtml> (consulté le 26 janvier 2011).

HUGON M.-A. (1984). "Situation et fonction des classes de perfectionnement dans l'enseignement français ". Revue française de pédagogie, $n^{\circ} 66$, p. 55-67.

LAURENT-COGNET J. (2005). “ La fonction d'auxiliaire de vie scolaire (intégration individuelle) est-elle une fonction originale? "La nouvelle revue de l'adaptation et de la scolarisation, $\mathrm{n}^{\circ} 30$, p. 115-123.

MAZEREAU P. (2005). « L'accompagnement, nouveaux modèles et nouveaux dispositifs. Quels modes de coopération professionnelle pour les enfants en situation de handicap ? " La nouvelle revue de l'adaptation et de la scolarisation, $\mathrm{n}^{\circ} 30$, p. 57-65.

MINISTĖRE DE L'ÉDUCATION NATIONALE (2008). "Formation des auxiliaires de vie scolaire". Circulaire $\mathrm{n}^{\circ}$ 2008-100 du 24 juillet 2008. Bulletin officiel de l'Éducation nationale, 31 juillet 2008, $\mathrm{n}^{\circ} 31$.

MINISTÈRE DE L'ÉDUCATION NATIONALE (2010). La scolarisation des élèves handicapés. Paris : Ministère de l'Éducation nationale. Disponible sur Internet à l'adresse : <http://www.education.gouv.fr/cid207/ la-scolarisation-des-eleves-handicapes.html> (consulté le 26 janvier 2011).

PERRENOUD P. (1993). « La division du travail pédagogique à l'école primaire ". In A. Henriot-van Zanten, É. Plaisance \& R. Sirota (dir.), Les transformations du système éducatif. Acteurs et politiques. Paris : L'Harmattan, p. 29-46.

PERRENOUD P. (1994). “Formation initiale des maîtres et professionnalisation du métier ». In P. Perrenoud, La formation des enseignants entre théorie et pratique. Paris : L'Harmattan, p. 175-196.

PHILIP C. (2009). “Présentation d'un projet de formation pour les AVS de l'académie de Créteil mis en œuvre par I'INS HEA entre 2004 et 2007 ". La nouvelle revue de l'adaptation et de la scolarisation, $\mathrm{n}^{\circ} 45$, p. 104-110.

PLAISANCE É. (2009a). «Conférence de consensus 2008. Scolariser les élèves en situation de handicap : pistes pour la formation ". Recherche et formation, $\mathrm{n}^{\circ} 61$, p. 11-40.

PLAISANCE É. (2009b). Autrement capables. École, emploi, société : pour l'inclusion des personnes handicapées. Paris : Autrement.

PUIG J. (2005). «Élèves et étudiants en situation de handicap ». Reliance, $\mathrm{n}^{\circ} 16, \mathrm{p} .61-66$. 
STIKER H., PUIG J. \& HUET O. (2009). Handicap et accompagnement. Nouvelles attentes, nouvelles pratiques. Paris : Dunod.

TOULLEC-THÉRY M. \& NÉDÉLEC-TROHEL I. (2008). "Étude et catégorisation de pratiques effectives entre professeurs et auxiliaires de vie scolaire (AVS) à l'école primaire ". Alter. Revue européenne de recherche sur le handicap, vol. $2, n^{\circ} 4$, p. 337-358.
TOULLEC-THÉRY M., DUJARDIN D., NÉDÉLEC-TROHEL I. et al. (2009). " Un projet partenarial en Ille-et-Vilaine pour la professionnalisation des auxiliaires de vie scolaire ". La nouvelle revue de l'adaptation et de la scolarisation, $\mathrm{n}^{\circ} 45$, p. 116-127.

UNION NATIONALE POUR L'AVENIR DE L'INCLUSION SCOLAIRE, SOCIALE ET ÉDUCATIVE (2008). Enquête nationale de I'UNAISSE sur les conditions de travail des AVS/EVS. Disponible sur Internet à l'adresse : $<$ http://unaisse.free.fr/images/enquete_academie/ Tableau_depouillement_Enquete_unaisse STRASBOÜRG.pdf> (consulté le 26 janvier 2011). 